\title{
Stretch Properties of Weft Knitted Fabrics
}

\author{
By Kentaro Kawasaki and Takayuki Ono, Members, TMSJ \\ Chori Co., Ltd., Higashiku, Osaka
}

Based on the Journal of the Textile Machinery Society of Japan, Transactions, Vol.19, No.4, T112 - 117 (1966-4)

\begin{abstract}
To clarify the relationship between the stretch properties of weft knit fabrics and their geometrical shape factors, a comparative measurement has been made of the stress-strain behaviors of such fabrics made of spun yarn and stretch yarn.

Results:

(1) The factors affecting the stretch of these fabrics are neither their loop-length, loop density nor the course wale ratio. None of these factors themselves affect it. Only their cover factor $d / l$ does ( $d$ : apparent diameter of yarn; $l$ : apparent loop length of fabrics).

(2) There is one master curve measured on fabrics knit in the same construction and of the same raw yarn but of different loop lengths.

Every stress-strain curve mentioned above can reach the master curve so iong as it shifts along the strain-axis for the distance given by the experimental constant and cover factor.

(3) It is suggested that the deformation of knit fabrics caused by extension be considered against the master curve obtained from the above shifting method in connection with its stressstrain properties, because the said behavior depends only upon the raw material and the knit construction, irrespective of the density of knitted fabrics.
\end{abstract}

\section{Introduction}

There have been several published studies dealing with the geometrical shape factor of weft knitted fabrics, but hardly any on their stretchability, except Fletcher and Robert's, ${ }^{[1]}$ which looks into the relationship between the maximum elongation, the residual strain and the cover factor $d / l$ ( $d$ : apparent diameter of yarn; $l$ : apparent loop length of fabrics) of cotton weft knitted fabrics.
This article tries to clarify the relationship between the geometrical shape factor and the stretch property of interlock double knitted fabrics and compare in stretchability stretch-yarn knitted fabrics and spun yarn knitted fabrics.

We have measured the stress-strain curve of interlock double knitted fabrics made of the different constitutions as shown in Table 1, looked into the relation between the maximum elongation (elongation under a constant load)

Table 1 Jergy Fabrics Used as Specimens

o Stretch jergy ( $70 \mathrm{~d}(24 \mathrm{f} / 2$ false nylon yarn, interlock)

\begin{tabular}{ccccccc}
\hline $\begin{array}{c}\text { Specimen } \\
\text { numbers }\end{array}$ & $\begin{array}{c}\text { Knitting } \\
\text { machine } \\
\text { (gauges) }\end{array}$ & $\begin{array}{c}\text { Loop } \\
\text { length } \\
(\mathrm{cm})\end{array}$ & $\begin{array}{c}\text { Density } \\
\text { Nale } \\
\text { wa) }\end{array}$ & $\begin{array}{c}\text { course } \\
\text { (C) }\end{array}$ & & \\
\hline 102 & 13 & 0.45 & 31 & 39 & 1209 & 0.78 \\
103 & 16 & 0.35 & 34 & 47 & 1598 & 0.72 \\
104 & 16 & 0.31 & 35 & 55 & 1925 & 0.63 \\
105 & 20 & 0.37 & 34 & 43 & 1462 & 0.78 \\
107 & 20 & 0.31 & 37 & 51 & 1887 & 0.73 \\
108 & 20 & 0.28 & 38 & 59 & 2242 & 0.65 \\
109 & 24 & 0.36 & 35 & 45 & 1575 & 0.78 \\
110 & 24 & 0.30 & 37 & 55 & 2035 & 0.69 \\
\hline
\end{tabular}

o Spun jergy

(1/48 acrylic spun yarn, interlock)

\begin{tabular}{ccccccc}
$\begin{array}{c}\text { Specimen } \\
\text { numbers }\end{array}$ & $\begin{array}{c}\text { Knitting } \\
\text { machine } \\
\text { (gauges) }\end{array}$ & $\begin{array}{c}\text { Loop } \\
\text { length } \\
(\mathrm{cm})\end{array}$ & $\begin{array}{c}\text { Density } \\
\text { No. of } \\
\text { wale } \\
\text { (W) }\end{array}$ & $\begin{array}{l}\text { No. of } \\
\text { course } \\
\text { (C) }\end{array}$ & $\mathbf{C} \times \mathbf{W}$ & W/C \\
\hline 111 & 13 & 0.44 & 27 & 29 & 783 & 0.92 \\
112 & 13 & 0.43 & 24 & 29 & 696 & 0.81 \\
113 & 16 & 0.31 & 33 & 45 & 1485 & 0.73 \\
114 & 16 & 0.29 & 32 & 47 & 1504 & 0.68 \\
117 & 20 & 0.32 & 34 & 37 & 1258 & 0.91 \\
118 & 20 & 0.30 & 36 & 43 & 1548 & 0.85 \\
120 & 24 & 0.29 & 37 & 42 & 1554 & 0.89 \\
\hline
\end{tabular}


and the various shape factors so as to substantiate Fletcher's theory, and examined the relation between the stress-strain curve upto the maximum elongation and the cover factors.

\section{Specimens and Method of Experimenting}

Two kinds of interlock double knitted fabrics (made of stretch and spun yarns of the same fiber but different counts) which were knitted by a circular knitting machine, 13-24 gauge, scoured and dyed by the ordinary method, were used as the specimens.

The yarns which were used for the two kinds of fabrics are as follows:

Stretch yarn: Toyo Rayon Co.'s "Woollie Nylon" (Trade name) nylon 6 false twist $70 \mathrm{~d} / 2,11 \mathrm{~d} / 2$.

Spun yarn: Toyo Rayon Co.'s "Torelon" (Acryl 100\% spun yarn) $1 / 36,1 / 48,1 / 52$.

(See Tables 1 and 2).

Table 1 shows specimens made of the same yarn $(70 \mathrm{~d} / 2$, $1 / 48$ ) and knit by varying the gauge of the knitting machine and course density. (knit fabrics made of stretch yarn will hereinafter be referred to as "stretch jergy"; knitted fabrics made of spun yarn, as "spun jergy.")

The stress-strain relation was measured with Instron Type Tester (Toyo Seiki's TENSILLON III) by the strip method (specimens were $5 \mathrm{~cm}$ wide and $10 \mathrm{~cm}$ long) at a cross head speed of $100 \mathrm{~mm} / \mathrm{min}$. The test was made at $20-25^{\circ} \mathrm{C}, 60-70 \%$ R.H. and each value is the average of 3 to 5 measured values.

The elongation test here was made in the course direction. The loop density of knitted fabrics was read with the naked eye with the aid of magnifying-glass (an enlarger). The loop length was calculated from the number of loops and yarn length measured under a constant load $(100 \mathrm{mg} / \mathrm{d})$ of deknit yarn. The diameter of yarn $(d)$ was caluculated from yarn denier and counts.

\section{Results of Measurement and Observation}

3-1. Relation between Stretchability of Knitted Fabrics and the Shape Factor.

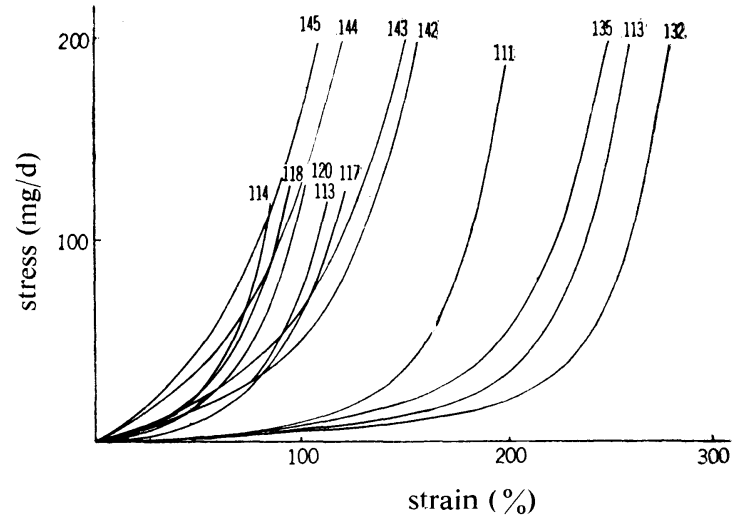

Fig. 1 Stress-strain curve of spun jergy

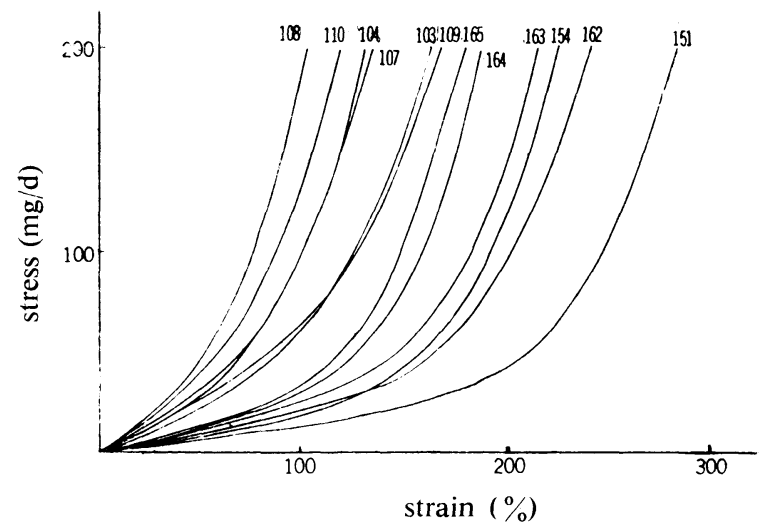

Fig. 2 Stress-Strain curve of stretch jergy

Figs. 1 and 2 show the stress-strain curves of spun jergy and stretch jergy. To reduce the effect of density of knitted fabrics, the stress axis was converted into stress per denier by dividing the load by the total denier within the width of the specimen (number of courses $\times 2$ (both surfaces of the specimen $) \times$ (denier of yarn used $)$.

Table 2 Jergy Fabrics Used as Specimens (2)

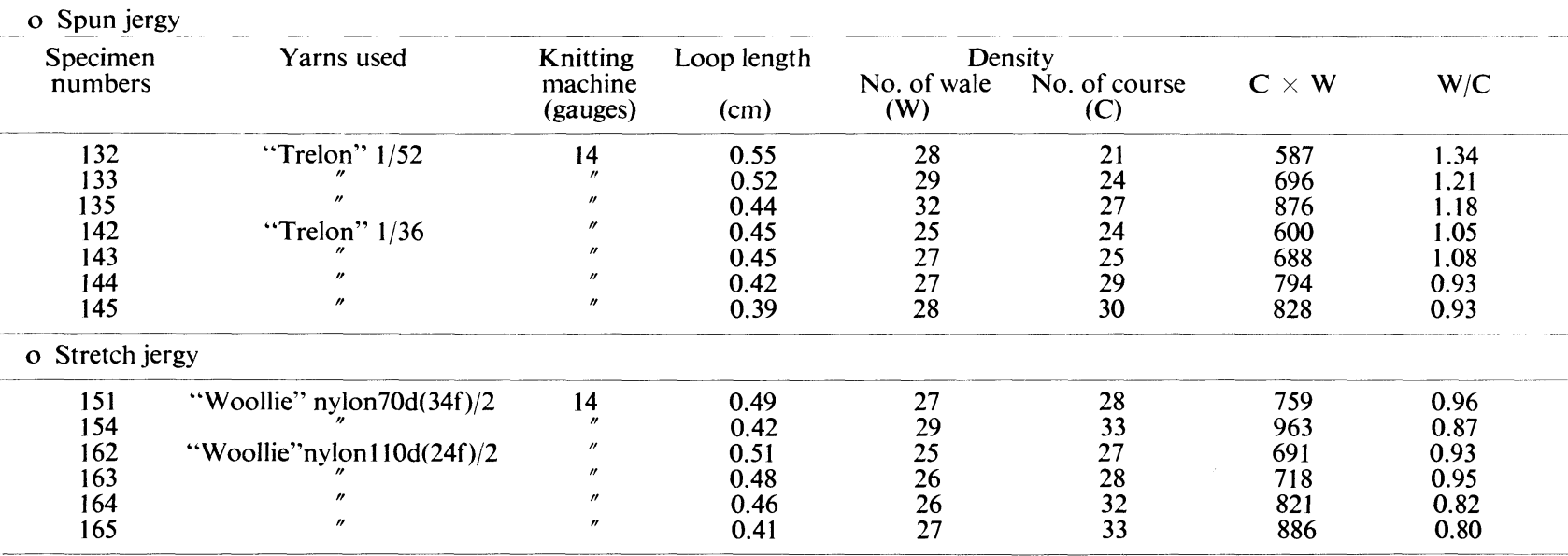


To clarify the relation between the stretchability of knitted fabrics and the geometric shape factor, we measured, as a representative value of stretch of knit fabrics, the elongation under a constant load of $100 \mathrm{mg} / \mathrm{d}$ (this elongation will be referred to hereinafter as "stretch"), and examined the correlation between stretchability and $w / c$ which is considered to be the factor of the following three; loop density $c / w$ (number of courses per inch $\times$ number of wale per inch), loop length $(l)$ and the loop shape.

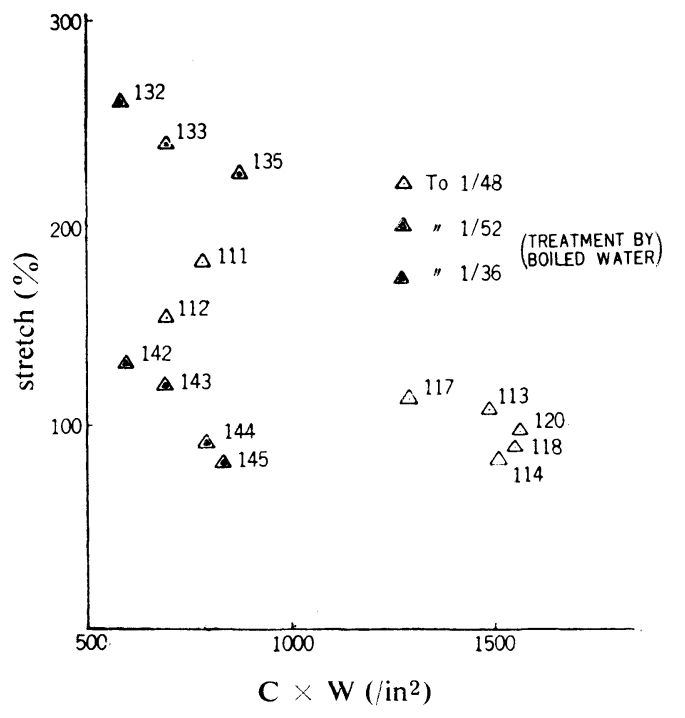

Fig. 3 Relation between stretch of spun jergy and $C \times W$

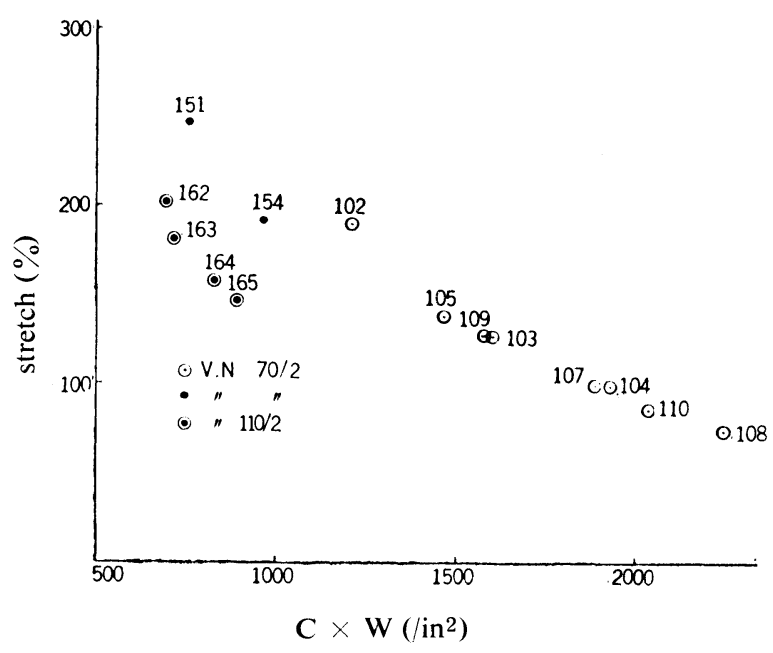

Fig. 4 Relation between stretch of jergy stretch and $\mathrm{C} \times \mathrm{W}$

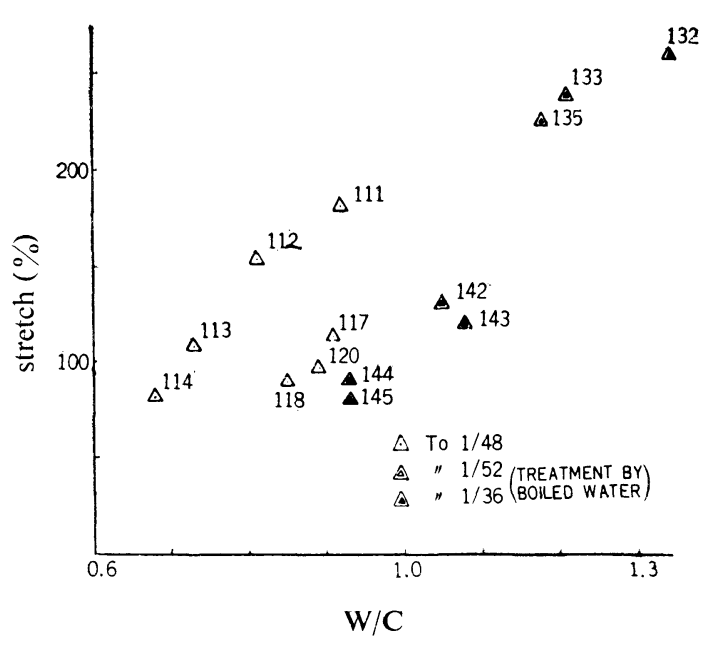

Fig. 5 Relation between stretch of spun jergy and W/C

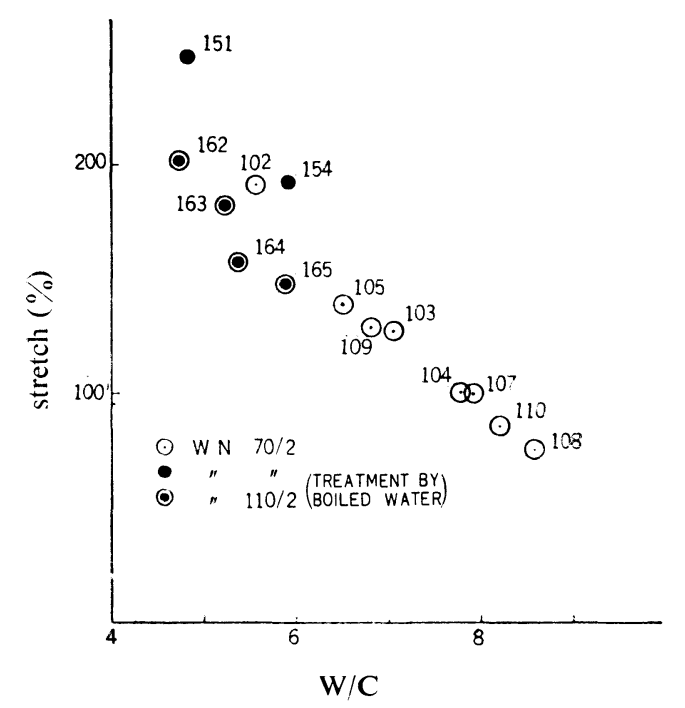

Fig. 6 Relation between stretch of stretch jergy and W/C

Figs.3-6 show the relation between stretchability and $c \times w$ or $w / c$. It is clear from these figures that the stretchability of knit fabrics has hardly any correlation to the above-mentioned shape factor, irrespective of the $c \times w$ of stretch jergy.

The authors examined the relation between the cover factor of knit fabric $d / l(d$ : denier of yarn) and its stretch in the light of Fletcher's theory. To explain the cover factor $d / l$, we have to cite Munder's theory. It is generally believed that there is the following relation between loop density $N$ of a knit fabric and its loop length if the fabric is plain knit :

$$
\begin{aligned}
& N=k / l^{2} \quad \ldots \ldots \ldots \ldots \ldots \ldots \ldots \\
& k: \text { experimental constant }
\end{aligned}
$$




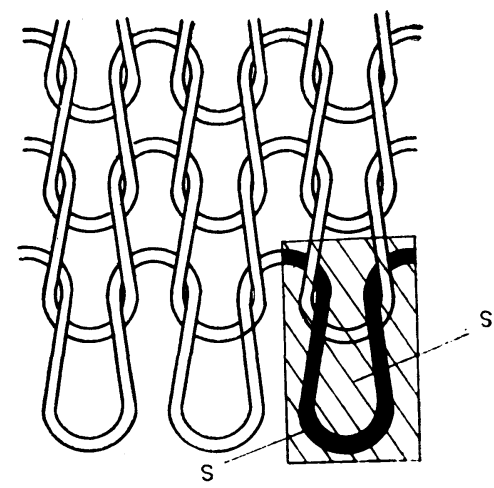

Fig. 7 Cover factor of knitted fabric

The tenability of eq.(1) to $1 \times 1$ rib, interlock, pique knit fabrics, as well as to plain knitted fabrics, is proved by T.S. Nutting, et al. Cover factor $(k)$ of knitted fabrics shows their density, as the model in Fig. 7 illustrates. It is defined as a ratio of yarn-occupied area $(s)$ to area $(S)$ which one loop construction occupies. Therefore,

$$
\begin{aligned}
& k=s / S \\
& N=l / s=k / l^{2}
\end{aligned}
$$

Accordingly,

$$
\begin{array}{r}
k=(l \times d) k / l^{2} \\
k d / l \propto d / l \quad . .
\end{array}
$$

This equation, however, is applicable only to knitted fabrics made of spun yarn which has hardly any stretch property, not to knit fabrics made of stretch yarn. In knitted fabrics, the yarn is relaxed, loop length $(l)$ decreases and the diameter of yarn $(d)$ increases, of course.

Assuming the apparent loop length to be $\left(l^{\prime}\right)$ and the apparent yarn diameter to be $\left(d^{\prime}\right)$, the cover factor $\left(k^{\prime}\right)$ of stretch yarn is:

$$
k^{\prime} \propto d^{\prime} / l \text { ' }
$$

Assuming the volume of the stretch yarn dose not change with the shrinkage in the length of yarn,

$$
\begin{gathered}
\left(d^{\prime} / d\right)^{2}=l^{\prime} / l \quad \ldots \ldots . \\
k^{\prime} \propto d^{\prime} / l=d / l^{\prime} \times \sqrt{l / l},
\end{gathered}
$$

The apparent diameter $\left(d^{\prime}\right)$ is difficult to measure, and the apparent loop length $(l)$ also is difficult to measure exactly, but it is possible to measure them approximately by the following:

Draw out yarns from a knit fabric, taking care to avoid giving exccessive tension to them, put them softly on a glass plate and cover them softly with another glass plate. A specimen is thus made. The length of the crimped yarn was measured along the line marked on the center of the yarn, and then averaged to obtain the apparent length $\left(l^{\prime}\right)$.

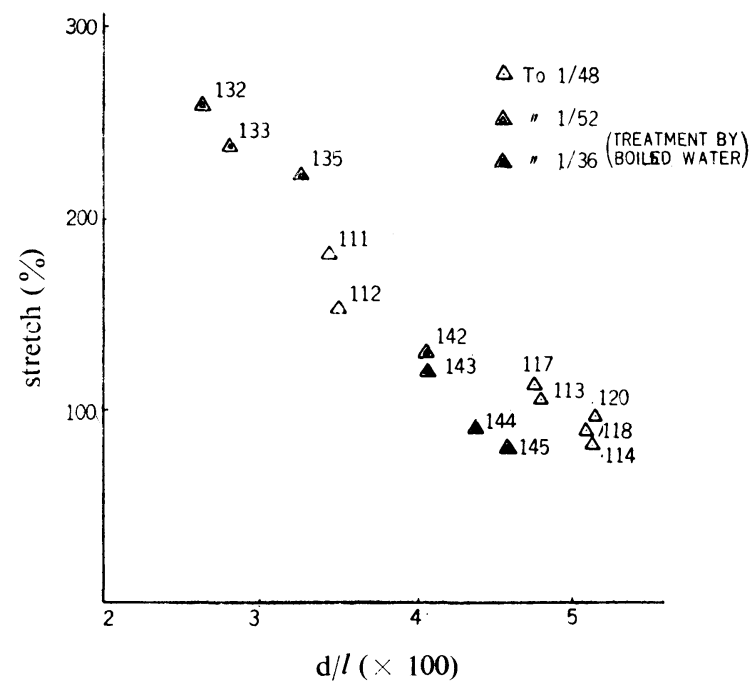

Fig. 8 Relation between stretch of spun jergy and $d / /$

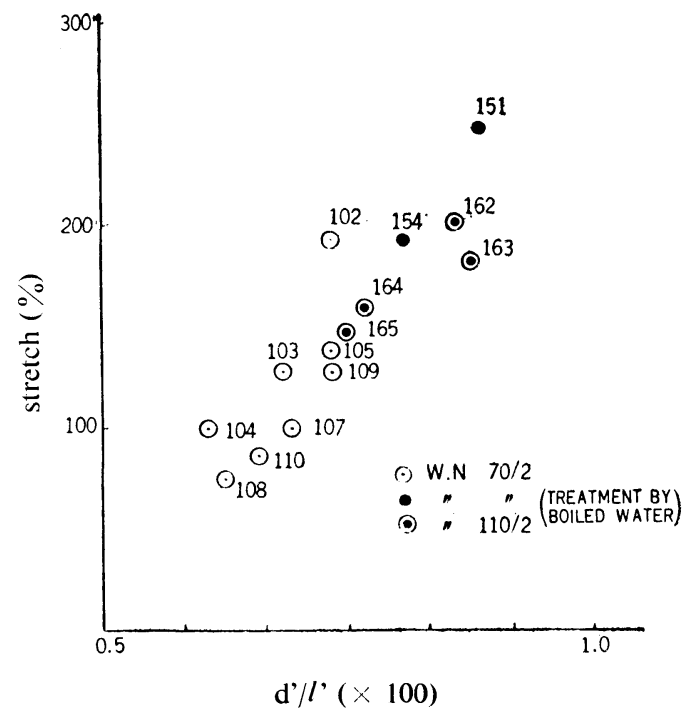

Fig. 9 Relation between stretch of stretch jergy and $\mathrm{d}^{\prime} / l$,

Fig. 8 shows the relation between the stretch and cover factor in spun jergy. Fig 9 shows the relation between the stretch and the cover factor calculated by eqs. (4), (5) and (6) and by using (1) measured by the above-mentioned method. The figures prove that the stretch of knit fabrics is affected more by the cover factor than by the yarn diameter, loop length, loop density or the shape of the loop. 3-2. Overlapping of Stress-strain Curves.

We find that, in each of knit fabrics made of the same fiber, not only the maximum elongation under a constant load but also the stress-strain curve across the whole stretched area is related to the cover factor. As shown in 


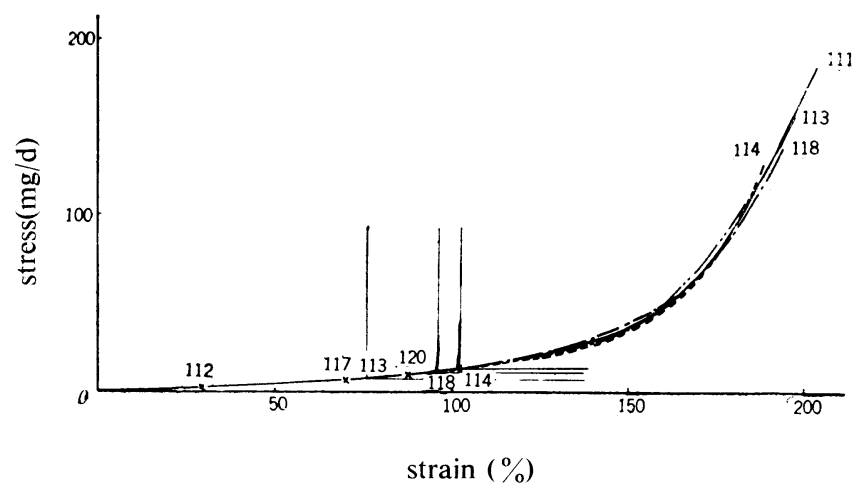

Fig. 10 Overlapping of stress-strain of spun jergy

Figs. 10 and 11 , each curve can be superimposed with a standard curve over the whole curve by shifting up or down, to right or left.

Assuming that the elongation be $x$, the stress at the elongation $x$ be $f(x)$, another overlapped curve be $g(x)$, and the amount of the parallel transfer for overlapping be $x_{o}$, then each curve can be expressed by the same function, as the following equation shows:

$$
\begin{aligned}
g(x) & =f\left(x+x_{o}\right)-f\left(x_{o}\right) \\
& =F\left(x, x_{o}\right) \ldots \ldots \ldots \ldots
\end{aligned}
$$

Let "transfer elongation" be the difference $\left(x_{0}\right)$ in elongation between the curve used as a standard and the curve transferred parallel for overlapping (the transfer amount of the origin) and each curve can be superimposed by adding the transfer elongation to the original elongation.

The stretch under a certain load is in inverse proportion to $d / l$. Therefore, assuming that the transfer elongation is also in inverse proportion to $d / l$, then the relation between the stress-strain curve and the cover factor can be clarifed.

Figs. 13 and 14 show the relation between the transfer elongation and the cover factor in stretch and spun jergies which are made of yarns of various deniers. There is clearly a fairly good correlation between the cover factor and the transfer elongation.

As Fig. 15 shows, with the values of the transfer elongation along the axis of ordinates and the values of $d / l$ along the axis of abscissas, the following linear equation can be shown:

$$
\delta=\beta(d / l)-\alpha
$$

Assuming that the abscissa of the point where the linear line and the axis of abscissas cross each other is $(d / l)$, the following equation is obtainable:

$$
\dot{o}=\beta\left\{(d / l)-(d / l)_{\mathrm{o}}\right\}
$$

$(d / l)_{0}$ being the value of $d / l$ when $\delta=0$, it is understood to be a coefficient determined by how the standard curve is chosen. In other words, transfer elongation $\delta$ can be expressed by (a) coefficient $\beta$ determined by the kind of the yarn and construction of the knit fabrics and (b) cover factor $(d / l)$.

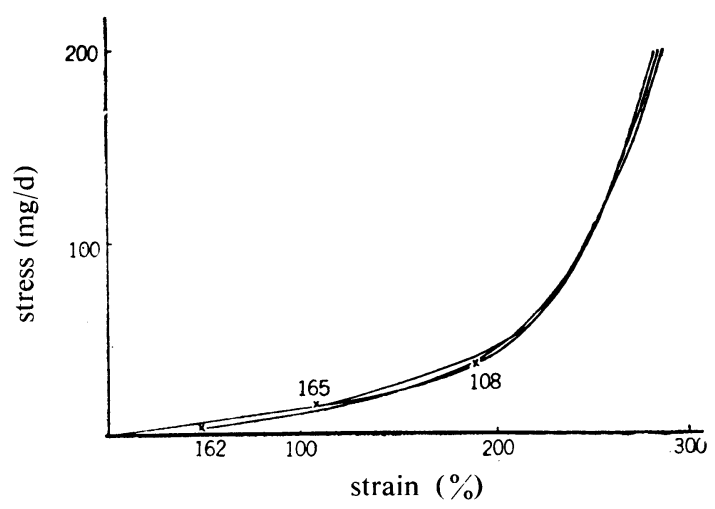

Fig. 11 Overlapping of stress-strain curves of stretch jergy

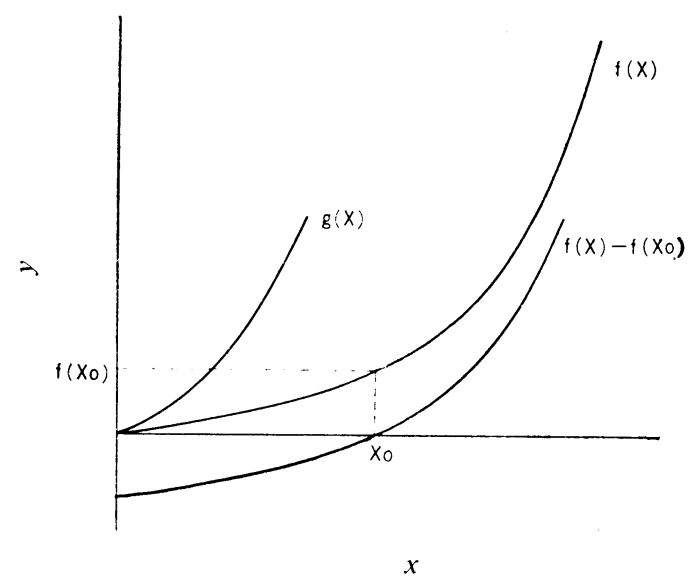

Fig. 12 Overlapping of stress-strain curves

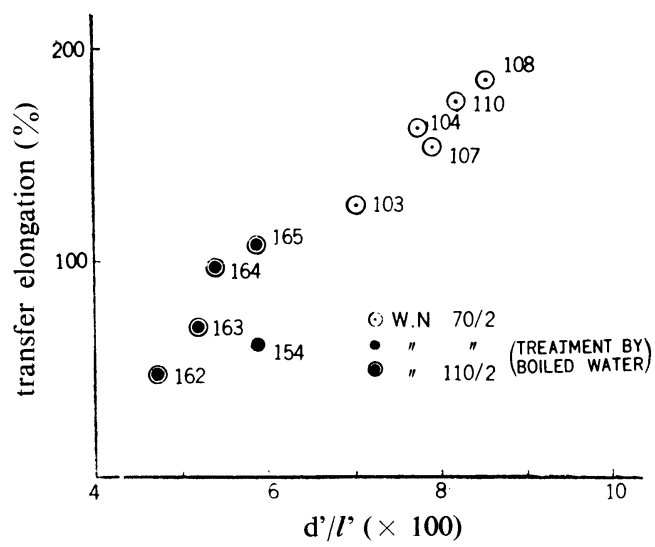

Fig. 13 Relation between transfer elongation of stretch yarn and d'/l' 


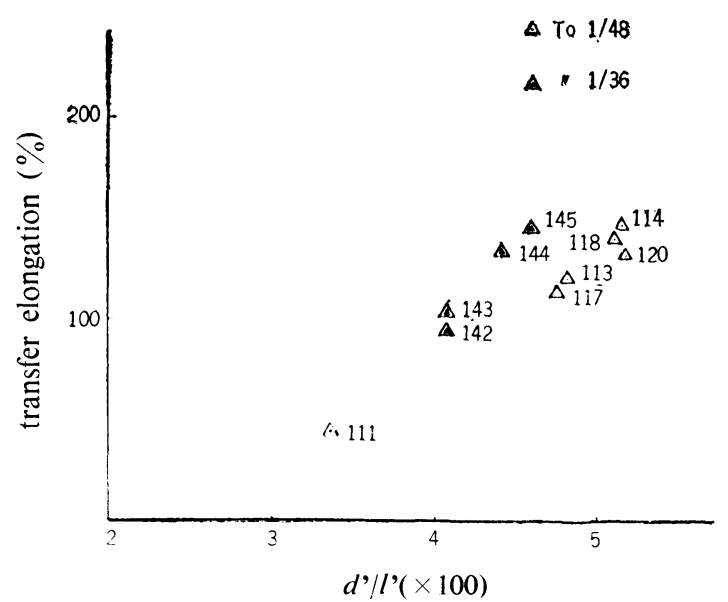

Fig. 14 Relation between transfer elongation of spun jergy and $d / l$

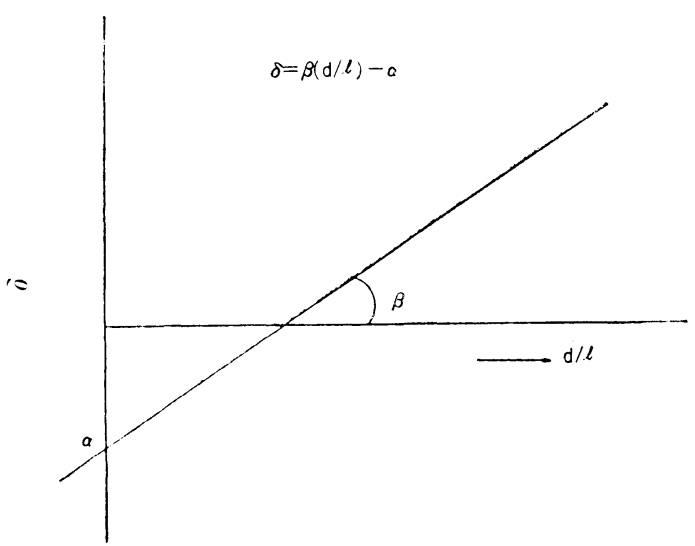

Fig. 15 Relation between transfer elongation and cover factor

Using constant $\beta$ obtained in Figs. 13 and 14, we obtained Figs. 16 and 17 by parallel shifting of the strain axis of various stress-strain curves in Figs. 1 and 2 by $(d / l)$. The amount of parallel transfer toward the stress axis for overlapping is smaller than the amount of transfer toward elongation. It can be made still smaller by shifting to the stress axis each of stress-strain curves rectified in the direction of the elongation by the use of the transfer stress calculated by the same method.

The curves are not always completely superimposed each other as shown in Figs.16 and 17. However, it can be expected that there exist "standard or Master stress-strain curve".

\section{Conclusions}

We have observed the stress-strain curves on interlock double knit fabrics made of stretch yarn and spun yarn. We have found that they can be represented by a master curve; and that the amount of transfer for the overlapping

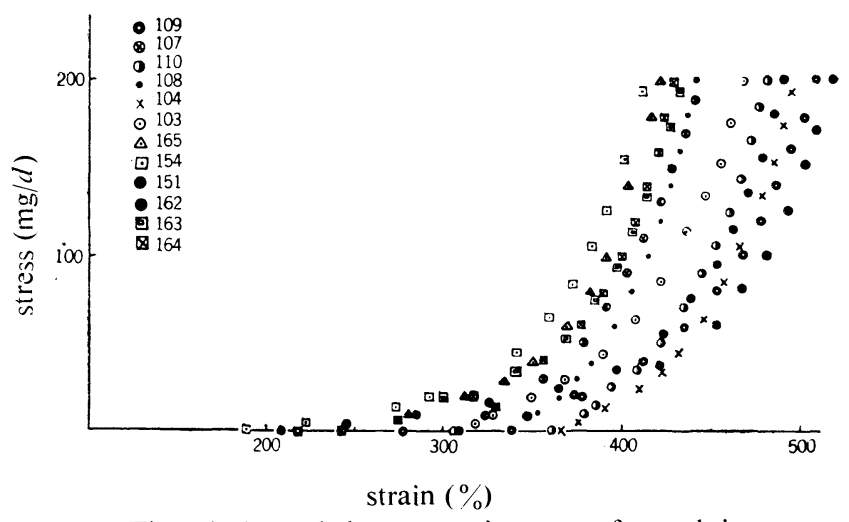

Fig. 16 Amended stress-strain curve of stretch jergy

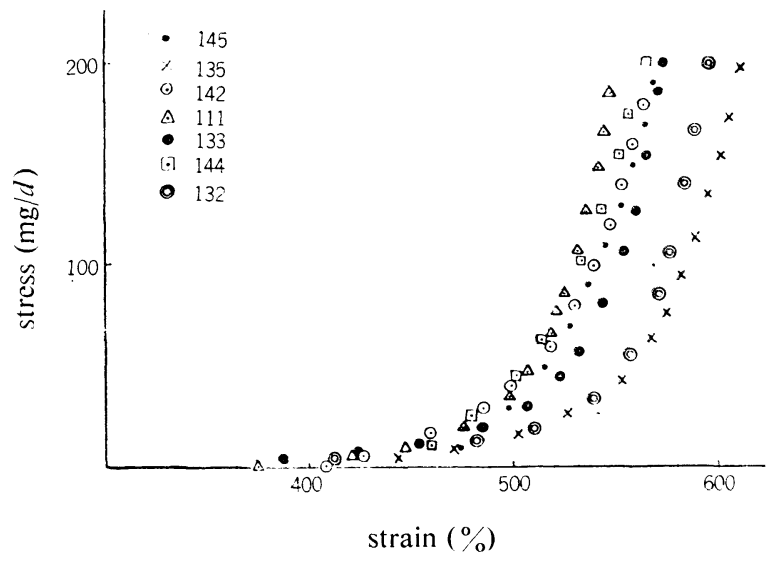

Fig. 17 Amended stress-strain curve of spun jergy

of curve can be determined only by the cover factor of knit fabric.

Accordingly, the deformation of knitted fabrics is affected by the same mechanism, irrespective of density, coarseness or stretch. The characteristics of the stress-strain of knitted fabrics had better be judged by observing the master curve after the effect of the cover factor is eliminated.

In this experiment, some deviations were noticed in the measured values of overlapping, the deviations were presumably caused by an unavoidable unreversible slippages among the loops.

If a knit fabric is elongated repeatedly and stabilized with less inter-yarn friction (for this purpose, elongation in water is perhaps a good idea) before measuring its stressstrain property, we can obtain an almost overlapped curve and a master curve.

We shall discuss at a later day the effects of the construction of knit fabrics and properties of yarns. 